\title{
AHP and Two-Envelope Paradox Resolution via Nonlinear Scaling
}

\author{
Stan Lipovetsky \\ Minneapolis, MN, USA \\ stan.lipovetsky@gmail.com
}

\begin{abstract}
Two Envelopes paradox presents a fascinating problem in probability and decision making. The player is presented with two envelopes and informed that one of them contains twice as much money as the other one. The player takes one of them without looking inside, and is then given the opportunity to change their mind and take the second envelope instead of the first one. Assuming that the $1^{\text {st }}$ envelope contains a value $A$, then the $2^{\text {nd }}$ one can have $2 \mathrm{~A}$ or $\mathrm{A} / 2$ with equal probability, and its expected value is the mean $1.25 \mathrm{~A}$, or $25 \%$ profit from switching envelopes. It is a great result, but we could denote the amount in the $2^{\text {nd }}$ envelope as $\mathrm{A}$ and repeat the derivation outcome $1.25 \mathrm{~A}$ already in $1^{\text {st }}$ envelope, so each of them is worth more than the other one. So, to switch or not to switch? - That is the question. As shown in this article, the ideas borrowed from the Analytic Hierarchy Process can help in resolving this paradox by transforming the ratio scale into the additive or logarithmic scales which correspond to application of the multiplicative utility function.
\end{abstract}

Keywords: Two-Envelope paradox, AHP, nonlinear scaling, transformation to shares and to logarithms, multiplicative utility function. 


\section{Introduction}

Among various renowned paradoxes related to the probability theory and statistics (for example, Gardner, 1982; Székely, 1986; Mosteller, 1987; Smullyan, 1997; Chang, 2012; Eckhardt, 2013), the Two-Envelopes paradox is maybe one of the most famous problems. It was proposed by Kraitchik (1953), and has been studied in multiple works based on probability theory and decision making, Bayesian evaluations, game theory and mathematical economics, logic and epistemology (see Linzer, 1994; Chalmers, 2002; Albers et al., 2005; Schwitzgebel and Dever, 2008; Falk and Nickerson, 2009; Abbott et al., 2010; Syverson, 2010; Markosian, 2011; McDonnell et al., 2011; Lee, 2013; Yi, 2013; Egozcue and Garcia, 2015). An extensive description of its different versions and trying to resolve the problem can be found on the web (Wikipedia, 2020, and the references within). In a lay description of the paradox's main version as a non-informed game, the player is presented with two envelopes and told that one of them contains twice as much money as the other envelope. The player takes one of them without looking inside, and then has the option to change their mind and take another envelope instead of the first one. It seems that without any information of the content the player can value both envelopes equally, so there is no reason to substitute one with another. However, assuming that the $1^{\text {st }}$ envelope contains a value $A$, then the $2^{\text {nd }}$ can have $2 A$ or $A / 2$ equally probable, and its expected value is the mean value

$$
(2 A+0.5 A) / 2=1.25 A,
$$

which is $25 \%$ profit from switching envelopes. It is a great result, but we could denote the amount in the $2^{\text {nd }}$ envelope as $A$ and repeat the derivation getting $1.25 A$ already in $1^{\text {st }}$ 
envelope, so each of them is worth more than the other one - The grass is always greener on the other side (Nalebuff, 1989). So, to switch or not to switch? - That is the question. To answer this request, we can apply some ideas from the Analytic Hierarchy Process (AHP) for resolving the problem via a nonlinear scaling approach. The reason for it is as follows. Measuring values in the second envelope are two times larger or smaller than in the first envelope, actually corresponds to the ratio scale. However, a ratio scale is not an additive, and if an addition is performed in the ratio scale it could easily produce unclear or strange results, exactly as it happens in the paradox of two envelopes. Resolution of this problem can be achieved by transforming the ratio scale into a scale which permits the operation of addition. Such a transformation can be done with the share and logarithm of the quotients. In such nonlinear scales the expected value equals zero, and the corresponding expected value in the original scale equals one, thus showing there is no gain in changing envelopes, and the paradox is dissolved. Let us describe these scales in more detail.

\section{Transformation of mutually reciprocal values into their shares}

The ratio scales are well-known in some multiple-criteria decision making (MCDM) methods. For example, the AHP widely used for solving problems of prioritization is based on the data elicited via pairwise quotients. Due to AHP founder Thomas Saaty $(1980,1994$, 1996) the pairwise comparisons among multiple criteria are made in the ratio scale of how many times one criterion is preferred over another one. If one criterion is $K$ times preferred over another criterion then the ratio of their preferences equals $K$. The value $K$ is measured by the natural numbers from 1 when the criteria are equal, 2 for a slight preference, etc., up to 9 of the overwhelming preference. If a preferences' ratio of the $i$-th to $j$-th item equals 
$K$ then the opposite preferences' ratio of the $j$-th to $i$-th item equals the reciprocal value $1 / K$. After eliciting the pairwise ratios from an expert, the matrix of the pairwise comparisons is built, with the elements $a_{i j}$ corresponding to the ratio of priorities of the $i$ th to $j$-th items. The matrix elements are reciprocally transposed, so $a_{i j}=1 / a_{j i}$, for instance, if $a_{i j}=2$ then $a_{j i}=0.5$, or if $a_{i j}=8$ then $a_{j i}=0.125$. The bigger values have a bigger impact in numerical estimations performed in the regular additive scale. The classical AHP solves the eigenproblem of such a matrix, and its first eigenvector serves as the vector of the items' priorities. The eigenvector priority estimation could be prone to possible errors in the data, and the results of priority estimation could become too sensitive because of the unbalanced impact of the bigger ratios and their too small reciprocal counterparts.

Lipovetsky and Conklin $(2002,2015)$ suggested transforming the matrix of pairwise ratios into the matrix of their shares and showed that such a matrix can be obtained via the Markov chain modeling. Implying the idea of reciprocal numbers' conversion into the balanced additive scale for the problem of two envelopes, let us consider the transformation of a value $K$ and $1 / K$ into their shares $S_{K}$ and $S_{1 / K}$, respectively. It can be done by the following formulae:

$$
S_{K}=\frac{1}{1+\frac{1}{K}}=\frac{K}{K+1} ; \quad S_{1 / K}=\frac{1}{1+\frac{1}{1 / K}}=\frac{1}{K+1} ; \quad S_{K}+S_{1 / K}=1 .
$$

For an example of $K=2$ and $1 / K=0.5$, the shares of these values by (2) are:

$$
S_{K=2}=\frac{1}{1+\frac{1}{2}}=\frac{2}{3} ; \quad S_{\frac{1}{K}=0.5}=\frac{1}{1+\frac{1}{1 / 0.5}}=\frac{1}{3} ; \quad S_{2}+S_{0.5}=1 .
$$

For $K=1$, as in the first original envelope's value, the transformation (2) yields the share

$$
S_{K=1}=\frac{1}{1+\frac{1}{1}}=\frac{1}{2} .
$$

The deviations $D$ of the share values (2) from the basic level (4) are as follows: 


$$
D S_{K}=\frac{K}{K+1}-\frac{1}{2}=\frac{K-1}{2(K+1)} ; \quad D S_{\frac{1}{K}}=\frac{1}{K+1}-\frac{1}{2}=\frac{1-K}{2(K+1)} ; \quad D S_{K}+D S_{1 / K}=0,
$$

so the centered shares' total equals zero.

For the example with $K=2$, the deviations of the shares (3) from the basic level calculated by (5) are:

$$
D S_{K=2}=\frac{2-1}{2(2+1)}=\frac{1}{6} ; \quad D S_{\frac{1}{K}=0.5}=\frac{1-2}{2(2+1)}=-\frac{1}{6} ; \quad D S_{K=2}+D S_{\frac{1}{K}=0.5}=0 .
$$

The expected utility with equal probabilities, or the mean value of the shares calculated by the nonlinear scale, due to the last relation in (3) is:

$$
0.5 S_{2}+0.5 S_{0.5}=\frac{1}{2}
$$

which equals the basic level (4). It coincides with the utility in the first envelope, and no substitution of the envelopes changes this utility. Therefore, in the adequate scale of shares for measuring utility, the paradox dissolves.

\section{Multiplicative utility and logarithmic transformation}

The ratio scales for paired comparisons among many items are used with other MCDM methods as well, for example, in the multiplicative AHP (Saaty and Vargas, 1984, 1994; Lootsma, 1993, 1999; Lipovetsky and Tishler, 1999) where the pairwise ratios are transformed by logarithm, so the reciprocal values $K$ and $1 / K$ become opposite numbers:

$$
\log (K)=-\log (1 / K)
$$

These logarithms are equal by their absolute value, so they are comparable in the additive scale. Applications of additive and multiplicative modes and their comparison have been studied in various problems, for instance, in index analysis in economics, in mixed probability distributions, in SVD techniques (Lipovetsky and Conklin, 2005, 2006; Lipovetsky, 2009, 2013). Transforming to logarithmic scales corresponds to using a 
multiplicative utility function known by many works (for example, Keeney, 1974; Mehrez et al., 1988).

For $K=2$ and $1 / \mathrm{K}=0.5$, which corresponds to the two-envelopes problem, the expected utility with equal probabilities, or the mean value in the logarithmic scale (8), equals:

$$
0.5 \log (2)+0.5 \log \left(\frac{1}{2}\right)=0 .
$$

As $\log (1)=0$, the expected utility in the original scale equals 1 , which is the utility in the first envelope with the amount $A(1)$.

The same conclusion can be obtained if to estimate the expected utility not by the arithmetic mean but by the geometric mean which is an adequate measure for the multiplicative utility. The expected value for the multiplicative utility is defined by the weighted geometric mean, so in the two-envelope problem with equal probability of choice between the envelope with $2 \mathrm{~A}$ and with $0.5 \mathrm{~A}$ values it is:

$$
(2 A)^{0.5}(0.5 A)^{0.5}=\sqrt{(2 A)(0.5 A)}=A,
$$

which coincides with the value in the original envelope. Thus, no substitution of the envelope changes the original utility, and in the adequate logarithmic scale for measuring utility in the multiplicative mode the paradox is resolved.

Comparing the shares and logarithmic scale we can note that they behave very similarly and differ by a constant term depending on the base of logarithm. Table 1 presents examples of share and logarithmic transformations. The ratio $K$ in the first column of Table 1 is taken from 1 to 9 and the corresponding reciprocal values. The next two columns show the values of the centered shares (5) and logarithms (8) taken by the base 100. The last three columns present the quotient $\log /$ shares with values around 1 , the deviations values of log-shares, and the absolute deviation values of $\mid \log$-shares|. In the last two bottom rows 
there are the mean values and standard deviations (std) of the values in each column, and all of those are very small. The results are mostly the same for a larger span of the ratios up to 100 as well, with the corresponding reciprocal values.

Table 1. Share and logarithmic transformations of the ratio.

$\begin{array}{cccccc}\text { ratio K } & \text { Shares (5) } & \text { Logarithm (8) } & \text { log/share } & \text { log-shares } & \begin{array}{c}\text { abs(log- } \\ \text { shares) }\end{array} \\ 1 / 9 & -0.400 & -0.477 & 1.193 & -0.077 & 0.077 \\ 1 / 8 & -0.389 & -0.452 & 1.161 & -0.063 & 0.063 \\ 1 / 7 & -0.375 & -0.423 & 1.127 & -0.048 & 0.048 \\ 1 / 6 & -0.357 & -0.389 & 1.089 & -0.032 & 0.032 \\ 1 / 5 & -0.333 & -0.349 & 1.048 & -0.016 & 0.016 \\ 1 / 4 & -0.300 & -0.301 & 1.003 & -0.001 & 0.001 \\ 1 / 3 & -0.250 & -0.239 & 0.954 & 0.011 & 0.011 \\ 1 / 2 & -0.167 & -0.151 & 0.903 & 0.016 & 0.016 \\ 1 & 0.000 & 0.000 & & 0.000 & 0.000 \\ 2 & 0.167 & 0.151 & 0.903 & -0.016 & 0.016 \\ 3 & 0.250 & 0.239 & 0.954 & -0.011 & 0.011 \\ 4 & 0.300 & 0.301 & 1.003 & 0.001 & 0.001 \\ 5 & 0.333 & 0.349 & 1.048 & 0.016 & 0.016 \\ 6 & 0.357 & 0.389 & 1.089 & 0.032 & 0.032 \\ 7 & 0.375 & 0.423 & 1.127 & 0.048 & 0.048 \\ 8 & 0.389 & 0.452 & 1.161 & 0.063 & 0.063 \\ 9 & 0.400 & 0.477 & 1.193 & 0.077 & 0.077 \\ \text { mean } & 0.000 & 0.000 & 1.060 & 0.000 & 0.031 \\ \text { std } & 0.330 & 0.363 & 0.098 & 0.042 & 0.026\end{array}$

For a particular $K$, it is possible to choose such a base $x$ for the logarithmic transformation that the centered share value $D S_{K}(5)$ will be exactly equal to this logarithm (8):

$$
\log _{x} K=D S_{K} .
$$

For example, if $K=2$ as in the two-envelope problem, the centered share value equals $1 / 6$ (as in the formula (6) and in Table 1), and solving the equation (11) yields:

$$
x=K^{1 / D S_{K}}=2^{6}=64 .
$$


Due to (11) change of the ratio $K=2$ by the logarithm of the base 64 gives the same centered share $1 / 6=0.167$, leading to both scaling transformations coinciding by their altered values. Shares and logarithm values from Table 1 profiled by the ratio values are presented by the graphs in Figure 1, which shows that both curves behave very similarly. Dependence of the shares by the logarithm of the ratio values is shown in Figure 2, where we see that the dependence is very close to the linear. Indeed, the coefficient of pair correlation between the shares and logarithms equals 0.997 , which is a very high level of connection, and it does not depend on the base of logarithm.

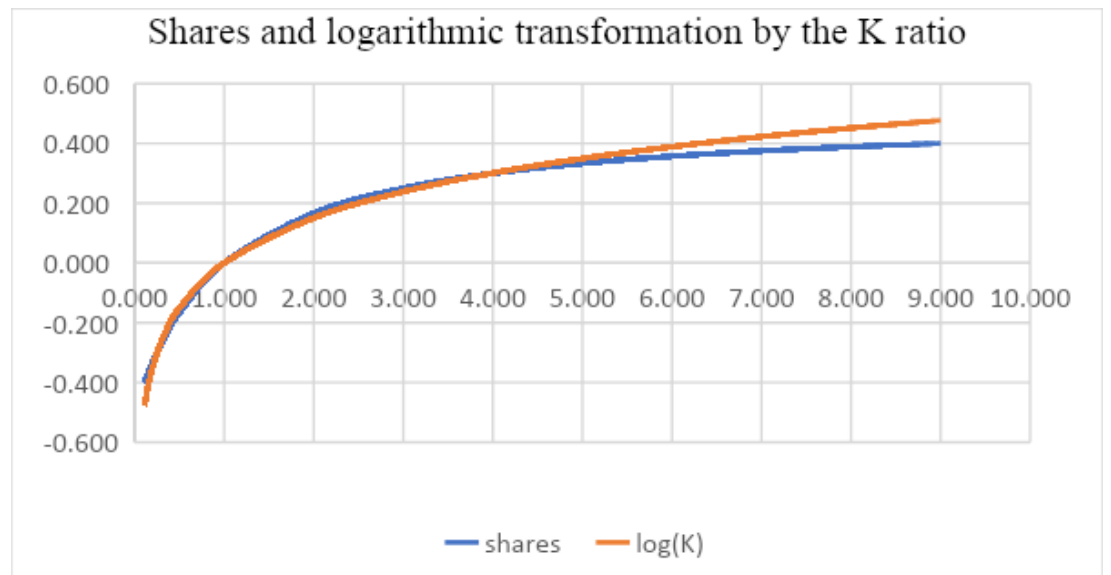

Figure 1. Shares and logarithm transformation profiled by the ratio values.

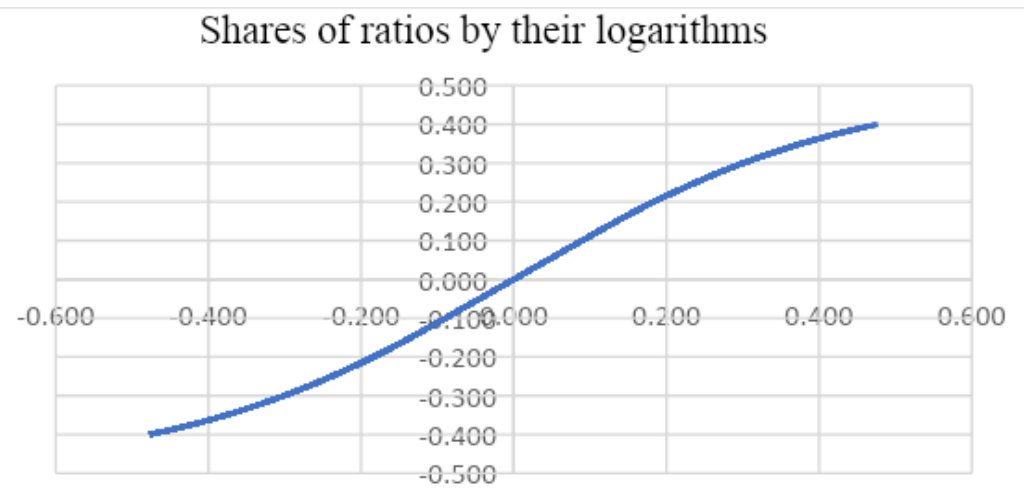

Figure 2. Shares by logarithms of the ratio values. 


\section{Summary}

This paper considers the two-envelope paradox and proposes to describe it in the nonlinear scales of the shares or logarithms of the values. Such kind of rescaling is known in multiplecriteria decision making methods, particularly, in the Analytic Hierarchy Process. It is shown that the two-envelope paradox can be easily dissolved if to use these scales, particularly, the logarithmic scale corresponding to the multiplicative utility with the geometric mean for the expected utility estimation. The reason for it is as follows: if in comparison with the original envelope value $\mathrm{A}$, the second envelope contains twice more or twice less, $2 \mathrm{~A}$ or $\mathrm{A} / 2$, then it means that the measurement is performed by the ratio scale with terms of multiplication 2 and 0.5 . But the ratio scale is not an additive scale, and if to use the arithmetic mean for the expected utility the result will be biased to the bigger value 1.25A. However, if to use the geometric mean, adequate to the ratio scale, then the result coincides with the original value A, and there is no paradox. Similar results are achieved with the scale of the shares of the multiplicative terms. Numerical comparison shows that both the shares and logarithmic scales are very close in a wide range of possible values of the ratios. The same fact that nonlinear scaling dissolves the oxymoronic conclusion of this paradox that each envelope is better to change to another one proves the correctness of using the nonlinear utility approach described in the paper. This technique helps to understand the two-envelope problem, solves its paradox, and can be useful in other applications of the multiple-criteria decision making.

\section{References}

Abbott D., Davis B.R., and Parrondo J.M. (2010) The two-envelope problem revisited, Fluctuation and Noise Letters, 9, 1-8.

Albers C.J., Kooi B.P., and Schaafsma W. (2005) Trying to resolve the two-envelope problem, Synthese, 145, 89-109. 
Chalmers D.J. (2002) The St. Petersburg Two-Envelope Paradox, Analysis, 62, 2, 155-157.

Chang M. (2012) Paradoxes in Scientific Inference, Chapman and Hall/CRC.

Eckhardt W. (2013) Paradoxes in Probability Theory, Springer, Chikago.

Egozcue M. and Garcia L.F. (2015) An optimal threshold strategy in the two-envelope problem with partial information, Journal of Applied Probability, 52, 1, 298-304.

Falk R. and Nickerson R. (2009) An inside look at the two envelopes paradox, Teaching Statistics, 31, 39-41.

Gardner M. (1982) Aha! Gotcha: Paradoxes to Puzzle and Delight, W.H. Freeman, New York.

Keeney R.L (1974) Multiplicative Utility Functions, Operations Research, 22, 1, 22-34.

Kraitchik M.B. (1953) Mathematical recreations, Dover, New York, NY.

Lee Ch. (2013) The Two-envelope Paradox: Asymmetric Cases, Mind, 122, 485, 1-26.

Linzer E. (1994) The Two Envelope Paradox, The American Mathematical Monthly, 101, $5,417-419$.

Lipovetsky S. (2009) Global Priority Estimation in Multiperson Decision Making, Journal of Optimization Theory and Applications, 140, 2009, 77-91.

Lipovetsky S. (2013) Additive and multiplicative mixed normal distributions and finding cluster centers, Int. J. of Machine Learning and Cybernetics, 4, 1, 1-11.

Lipovetsky S. and Conklin M. (2002) Robust Estimation of Priorities in the AHP, European Journal of Operational Research, 137, 110-122.

Lipovetsky S. and Conklin M. (2005) Singular Value Decomposition in Additive, Multiplicative, and Logistic Forms, Pattern Recognition, 38, 1099-1110.

Lipovetsky S. and Conklin M. (2006) Data Aggregation and Simpson's Paradox Gauged by Index Numbers, European Journal of Operational Research, 172, 334-351.

Lipovetsky S. and Conklin M. (2015) AHP Priorities and Markov-Chapman-Kolmogorov Steady-States Probabilities, International Journal of the Analytic Hierarchy Process, 7 (2), 2015, 349-363.

Lipovetsky S. and Tishler A. (1999) Interval estimation of priorities in the AHP, European Journal of Operational Research, 114, 153- 164. 
Lootsma F. (1993) Scale sensitivity in the multiplicative AHP and SMART, Journal of Multi-Criteria Decision Analysis, 2, 87-110.

Lootsma F. (1999) Multi-Criteria Decision Analysis via Ratio and Difference Judgement. Kluwer Academic Publishers, Dordrecht, Boston, London.

Markosian N. (2011) A Simple Solution to the Two Envelope Problem, Logos \& Episteme, II, 3, 347-357.

McDonnell M.D., Grant A.J, Land I., Vellambi B.N., Abbott D., and Lever K. (2011) Gain from the two-envelope problem via information asymmetry: on the suboptimality of randomized switching". Proceedings of the Royal Society A, 467, 2825-2851.

Mehrez A., Yuan Y., and Gafni A. (1988) Stable solutions vs. multiplicative utility solutions for the assignment problem, Operations Research Letters, 7, 3,131-139.

Mosteller F. (1987) Fifty Challenging Problems in Probability with Solutions, Dover.

Nalebuff B. (1989) The other person's envelope is always greener, Journal of Economic Perspectives 3, 171-81.

Saaty T.L. (1980) The Analytic Hierarchy Process. McGraw-Hill, New York.

Saaty T.L. (1994) Fundamentals of Decision Making and Priority Theory with the Analytic Hierarchy Process. RWS Publications, Pittsburgh, PA.

Saaty T.L. (1996) Decision Making with Dependence and Feedback: The Analytic Network Process. RWS Publications, Pittsburgh, PA.

Saaty T.L. and Vargas L.G. (1984) Comparison of eigenvalue, logarithmic least squares and least squares methods in estimating ratios, Mathematical Modelling, 5, 309-324.

Saaty T.L. and Vargas L.G. (1994) Decision Making in Economic, Political, Social and Technological Environment with the Analytic Hierarchy Process. RWS Publications, Pittsburgh, PA.

Schwitzgebel E. and Dever J. (2008) The two-envelope paradox and using variables within the expectation formula, Sorites, 20, 135-140.

Smullyan R. (1997) The Riddle of Scheherazade, and Other Amazing Puzzles, Ancient and Modern, Knopf, New York.

Syverson P. (2010) Opening Two Envelopes, Acta Analytica 25, 4, 479-498.

Székely G.J. (1986) Paradoxes in Probability Theory and Mathematical Statistics, Reidel/Kluwer. 
Wikipedia (2020) http://en.wikipedia.org/wiki/Two_envelopes_problem

Yi B.U. (2013) Conditionals and a Two-envelope Paradox, The Journal of Philosophy, 110, 5, 233-257. 